\title{
State of the journal
}

\section{Michael Lew ${ }^{1}$}

Published online: 9 November 2019

(c) Springer-Verlag London Ltd., part of Springer Nature 2019

This journal was started in 2012 because the emerging field of multimedia retrieval was growing quickly but did not have an archival journal. Around the same time, a new peer-reviewed conference, the ACM International Conference on Multimedia Retrieval (ICMR), had just started. Thus, it seemed to be the right time to start the first journal in the field of multimedia retrieval. Furthermore, it was well known that the computer science journals in related fields such as pattern recognition and computer vision and others often required $6+$ months to the first decision. Considering that the general science journals such as Nature and Science had first decision times of a month (or less), we knew that it was possible to perform the peer review well and more quickly, but it had not been implemented in any related journal from ACM, IEEE, Springer or Elsevier that we were aware of. These factors motivated our major starting goals: create a high quality archival journal for the field of multimedia retrieval and aim for an average time to the first decision of roughly 1 month.

The last 2 years have been remarkable for the International Journal of Multimedia Information Retrieval (IJMIR). Here is a brief summary of the state of the journal:

(1) Submissions We had a 60\% increase in submissions in the past 2 years, from 94 to 157 .

(2) Acceptance Our acceptance rate in 2018 was $17 \%$.

(3) Decision time Our time from submission to first decision in 2018 was 32 days.

(4) ICMR A special issue on the best papers from the ACM International Conference on Multimedia Retrieval (2018) was guest-edited by Benoit Huet, Qi Tian and Keiji Yanai.

The issue comprises the following papers:

Michael Lew

mlew@liacs.nl

1 Leiden University, Leiden, The Netherlands
- "Joint Embeddings with Multimodal Cues for VideoText Retrieval" by Niluthpol C. Mithun, Juncheng Li, Florian Metze, Amit K. Roy-Chowdhury,

- "Mining Exoticism from Visual Content with Fusionbased Deep Neural Networks" by Andrea Ceroni, Chenyang Ma and Ralph Ewerth,

- "Automatic Visual Pattern Mining from Categorical Image Dataset" by Hongzhi Li, Joseph G. Ellis, Lei Zhang and Shih-Fu Chang,

- "Multi-view Collective Tensor Decomposition for Cross-modal Hashing" by Limeng Cui, Jiawei Zhang, Lifang He and Philip S. Yu.

See https://link.springer.com/journal/13735/8/1.

(5) Research areas From the last author and reviewer survey, we are actively encouraging work in two related research areas: (1) the intersection of deep learning and multimedia understanding; and (2) understanding and addressing the human-in-the-loop in deep-learning-based retrieval. A special issue in this area is expected in summer 2020.

(6) Impact In terms of impact or average citations/paper, we have been in the top three multimedia journals worldwide for the past 4 years based on Scopus data.

Several noteworthy members of the editorial board have been the Senior Editors: Thomas S. Huang (UIUC) and Ramesh Jain (UCI). Not only have they helped guide the direction of the journal and provided timely feedback on proposals for surveys, special issues and other initiatives, but they have also been the organizers for special issues on important topics.

We are grateful to the support and cooperation from the ACM SIG Multimedia and the International MIR Society.

To all of our authors and reviewers, I would like to thank you for your support. You are the core of the community and this journal.

Publisher's Note Springer Nature remains neutral with regard to jurisdictional claims in published maps and institutional affiliations. 